\title{
Journal of Medical Microbiology \\ and Infectious Diseases
}

\section{Immunogenicity of the Recombinant Cryptococcus neoformans HSP70, a Potential Candidate for Developing an ELISA Kit}

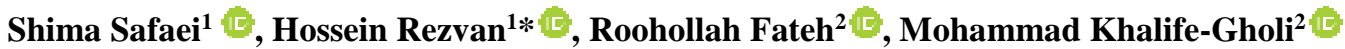 \\ ${ }^{1}$ Department of Pathobiology, School of Paraveterinary Sciences, BU-Ali Sina University, Hamadan, Iran; ${ }^{2}$ Department of \\ Microbiology and Immunology, School of Medicine, Qom University of Medical Sciences, Qom, Iran
}

\begin{abstract}
A R T I C L E I N F O
A B S T R A C T

Original Article

Keywords: Cryptococcus neoformans, Enzyme-Linked Immunosorbent Assay, Heat Shock Protein 70KD

Received: 25 Apr. 2020

Received in revised form: 26 Jul. 2020

Accepted: 27 Jul. 2020

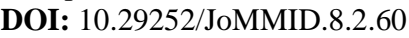

*Correspondence

Email: h.rezvan@basu.ac.ir

Introduction: Cryptococcus neoformans is an encapsulated fungal pathogen that causes life-threatening meningoencephalitis in immunocompromised patients. This yeast secretes several potent immunogenic proteins by secretory vesicular mechanisms, such as HSP70 chaperone. Methods: The PCR-amplified HSP70 gene was cloned into a PET-28a(+) expression vector. The purified recombinant HSP70 (rHSP70) was evaluated by western blotting using an anti-His Tag-HRP antibody and then used for immunization of a rabbit. The serum of the immunized rabbit was tested against the whole lysate of $C$. neoformans in ELISA. Results: The antibodies in the rabbit's serum recognized lysate of $C$. neoformans yeast. The highest antibody levels were achieved after the third booster injection. Conclusion: The rHSP70 showed to be a reliable candidate for the designing and development of an ELISA kit for early detection of cryptococcosis, and to screen a large number of specimens.
\end{abstract}

Tel: +988134227350

Fax: +988134227350

\section{INTRODUCTION}

Cryptococcus neoformans is a ubiquitous spherical encapsulated fungus found in soil, some trees, and bird excreta $[1,2]$. The infection occurs after inhalation and entrance of desiccated $C$. neoformans cells or fungal into the alveolar space. In the most immune-competent individuals, this infection either clears by the immune system or remains dormant within lung granulomas without evidence of clinical manifestation for an indefinite time until an immune imbalance leads to further development. In immunocompromised patients, the fungus disseminates in several parts of the body, especially the central nervous system. In severe cases, dissemination to the brain tissue leads to meningoencephalitis, which is the fatal form of the infection [1-5].

Cryptococcus neoformans has emerged as a leading cause of mortality in immunocompromised individuals, including $\mathrm{HIV}^{+} / \mathrm{AIDS}$ patients and organ transplantation recipients [1]. Worldwide, cryptococcal meningitis accounts for more than 1 million cases, with about 625,000 deaths annually [2, 4-7]. The prognosis of cryptococcosis depends on early diagnosis, primarily, when the load of the yeast is low. Early diagnosis can assist in the appropriate treatment of the patients and reducing the mortality of the infection [8, 9].

There are some limitations for current methods to diagnose cryptococcosis, including the need to a large number of yeast cells, the false-negative results in early stages of the infection due to the low burden of Cryptococcus yeasts and low sensitivity and specificity of the diagnostic tests $[2,9,10]$.

Humoral immunity is a crucial element of the host defense against $C$. neoformans. Studies showed that both the capsular polysaccharide and protein antigens of $C$. neoformans elicit antibody responses during infection [11].

The heat shock protein 70 (HSP70), a cellular chaperone, is synthesized in almost all organisms and plays a vital role during heat shock stress when the yeasts enter the host body. This protein is on the surface of the plasma cell membrane [12-15], and the host immune cells can recognize it [15]. This protein with a high concentration occurs in vesicular components of yeast cells and acts as a virulence factor [1517]. Hsp70 is one potent immunogenic protein that can stimulate humoral immune responses, particularly in pulmonary cryptococcosis $[13,14,17,18]$.

This study aimed to evaluate the immunogenicity of recombinant $C$. neoformans $\mathrm{HSP} 70$ for the development of a simple and sensitive method for the diagnosis of Cryptococcus infections.

\section{MATERIAL AND METHODS}

Fungal strain and culture media. The standard $C$. neoformans serotype A was obtained from Pasteur Institute 
and was grown on Sabouraud dextrose agar plates for $48 \mathrm{~h}$ at $30^{\circ} \mathrm{C}$.

Cloning of the C. neoformans hsp70 gene. The hsp70 gene was amplified by PCR using genomic DNA extracted from $C$. neoformans serotype A (PFCC No: 93-589 Cryptococcus neoformans). A NdeI site was introduced at the 5'-end of the forward primer (TGGCAAAATGACCGTGTCGAG) and an EcoRI site at 5 '-end of the reverse primer (TTAGTCGACCTCCTCAACGGA). The PCR product (2258 bp) was ligated into the $\operatorname{PBSK}(+)$ cloning vector (InsTAclone-PCR Cloning Kit, MBI Fermentas, Lithuania). The accuracy of the cloned sequence was confirmed by colony PCR and sequencing, as described elsewhere [10]. The cloned $h s p 70$ gene was subsequently subcloned into a PET-28a(+) expression vector (Qiagen, USA), and the recombinant plasmid was named pET-28a(+)-hsp70.

Expression of recombinant HSP70 (rHSP70). E. coli BL21 (Qiagen, USA) competent cells were transformed with pET-28a(+)- $h$ sp70 and inoculated in LB broth containing kanamycin $(50 \mu \mathrm{g} / \mathrm{ml})$. Expression of the recombinant protein was induced by the addition of $1 \mathrm{mM}$ IPTG (Isopropyl $\beta$-d-1-thiogalactopyranoside) at an optical density of 0.6 at $600 \mathrm{~nm}$. After $4 \mathrm{~h}$, the cells were harvested by centrifugation, and protein analysis of the whole lysate was performed by SDS-PAGE (Sodium dodecyl sulfatepolyacrylamide gel electrophoresis).

Purification and Western blotting of rHSP70. An amount of $50 \mathrm{ml}$ from induced bacteria was centrifuged, and the bacteria pellet was dispersed in $1 \mathrm{ml}$ volume of lysis buffer containing $10 \mathrm{mM}$ imidazole and homogenized by vortexing. The bacteria were lysed by sonication (six pulses with a power of $200 \mathrm{~W}$ for $10 \mathrm{~s}$ ), and soluble proteins were obtained by centrifugation at $13000 \mathrm{rpm}$ for $30 \mathrm{~min}$. Purification of the histidine-tagged rHSP70 was performed by immobilized metal affinity chromatography (IMAC). Non-specific binders were washed away using wash-buffer containing $30 \mathrm{mM}$ imidazole. Elution of rHSP70 was performed using elution-buffer containing $250 \mathrm{mM}$ imidazole. The eluted proteins were collected, passed from a centrifugal filter device (cut off $10 \mathrm{kDa}$ ), and stored at $-80^{\circ} \mathrm{C}$. The purified rHSP70 was evaluated by western blotting using an anti-His Tag-HRP antibody (Pierce, Rockford, USA).

Production of anti-rHSP70 antibodies in rabbit. A mature white New Zealand rabbit was immunized with the purified protein according to the protocol described by others (19). Briefly, immunization was conducted by intramuscular injection of $250 \mu \mathrm{g}$ purified HSP70 $(0.5 \mathrm{ml})$ with the same volume $(0.5 \mathrm{ml})$ of Freund's complete adjuvant. For the recall immunizations, the rabbit was re-injected with $125 \mu \mathrm{g}$ purified protein prepared in the same volume $(0.5 \mathrm{ml})$ of Freund's incomplete adjuvant, 4, 6, 8, and 10 weeks later. A serum sample before immunization was used as a negative control. Finally, two weeks after the last immunization, the rabbit blood was collected and serum separated.

ELISA. Two series of ELISA plates were prepared and coated with $50 \mu \mathrm{l}$ purified rHSP70 at a concentration of 2.5 $\mu \mathrm{g} / \mathrm{ml}$ in PBS and cell lysate of $C$. neoformans yeast as antigen layers. The ELISA plates were incubated overnight at $4^{\circ} \mathrm{C}$ and then washed three times with PBS-Tween 20, $0.05 \%$ (PBST). The coated wells were blocked with phosphate-buffered saline (PBS) containing $2.5 \%$ bovine serum albumin (BSA, Sigma) for $2 \mathrm{~h}$ at $37^{\circ} \mathrm{C}$. The immunized rabbit sera obtained at different time intervals post immunizations were added at $1 / 100$ dilutions. The plates were incubated for $2 \mathrm{~h}$ at $37^{\circ} \mathrm{C}$ and washed three times with PBST. Then, HRP-conjugated anti-rabbit IgG (Zist Fanavaran Sina, Iran) was added (1:500 dilution) as the secondary antibody, and the plates were incubated for 1 hour at $37^{\circ} \mathrm{C}$. After washing the plates with PBST, the substrate 3,3',5,5'-tetramethylbenzidine (TMB) was added, and optical densities were read $10 \mathrm{~min}$ later by ELISA reader (Biotek, USA) at $450 \mathrm{~nm}$, and $630 \mathrm{~nm}$ as the reference. Mean OD450 of duplicate wells was used for the preparation of the graph.

\section{RESULTS}

Amplification of the $h s p 70$ gene from C. neoformans genomic DNA revealed a single 2250 bp amplicon (Fig. 1), which was confirmed by sequencing and ligated into the expression vector pET-28a(+). After transformation into $E$. coli expression strain BL21, and induction with IPTG, a strong band of about $70 \mathrm{kDa}$ was observed on SDS PAGE (Fig. 2). The expression level was increased when IPTG induction time was increased from one to three hours (Fig. 2). The rHSP70 was purified using a native IMAC method, and a bright single band was observed in the elusion (Fig. 3). Western blot analysis confirmed the recognition of the purified protein with a monoclonal antibody against the histidine tag (Fig. 4). While rHSP70 was highly expressed by the bacteria, most of the protein was insoluble (Fig. 5). The low solubility resulted in low yield of the purified protein, as we only purified the soluble part of the expressed HSP70. To obtain a high amount of the protein, it must be purified under denaturing conditions and subsequently be refolded to obtain native structure. However, the refolding process may adversely affect native folding and antigenicity.

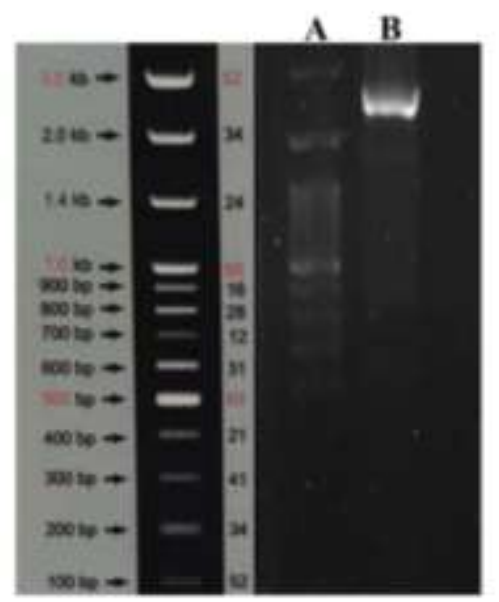

Fig. 1. Electrophoresis of PCR-amplified $h s p 70$ gene. A, DNA ladder; B, $h s p 70$ gene 

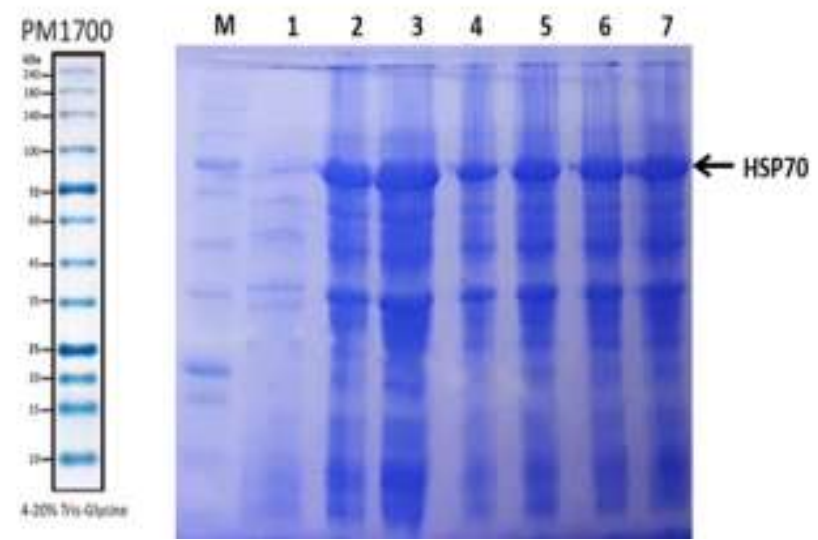

Fig. 2. Expression of HSP70 in different bacteria colonies on $12 \%$ SDS-PAGE gel. M, protein molecular weight marker; lane 1, sample before IPTG induction; lanes 2, 4, and 6, bacteria colonies, 1, 2 and 3, one hour after IPTG induction; lanes 3, 5 and 7 , colonies 1,2 , and 3 , four hours after IPTG induction

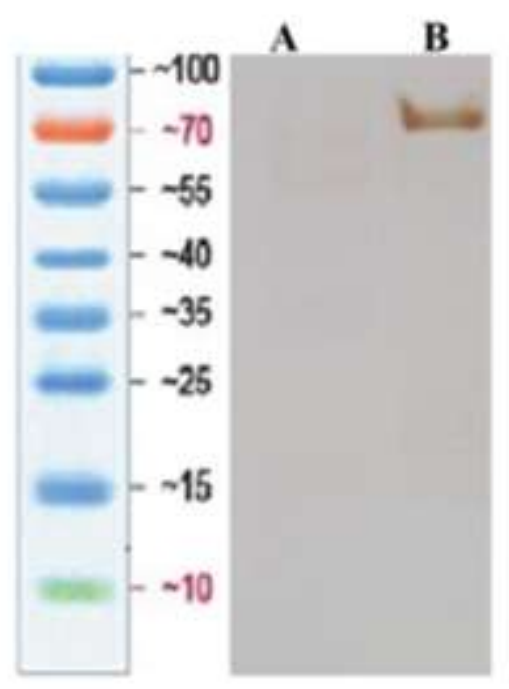

Fig. 4. Western blotting of Histidine-tagged rHSP70 using a monoclonal antibody against histidine tag. Lane A, protein ladder; lane B, rHSP70 protein

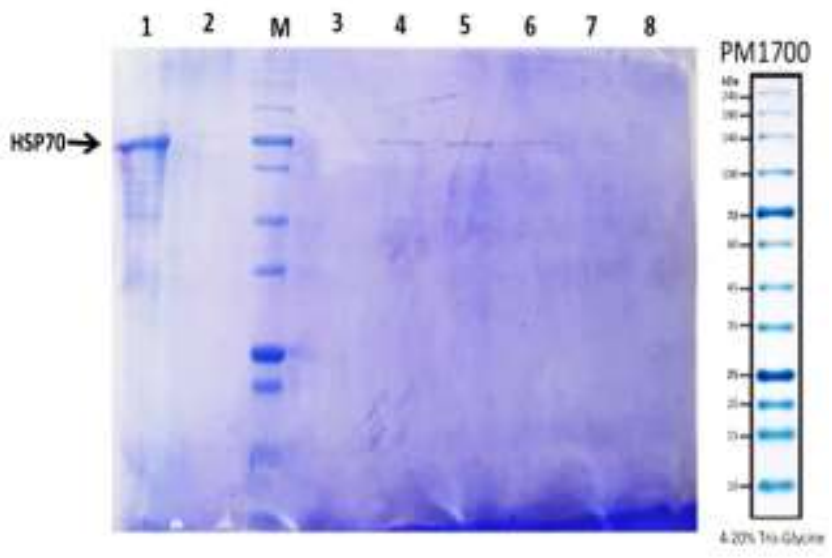

Fig. 3. Analysis of purification of rHSP70. Lane 1, soluble cell lysate before purification; lane 2, flow-through; lane M, protein molecular weight marker; lane 3 , washed away proteins; lanes 4-7, eluted proteins; lane 8, MES buffer

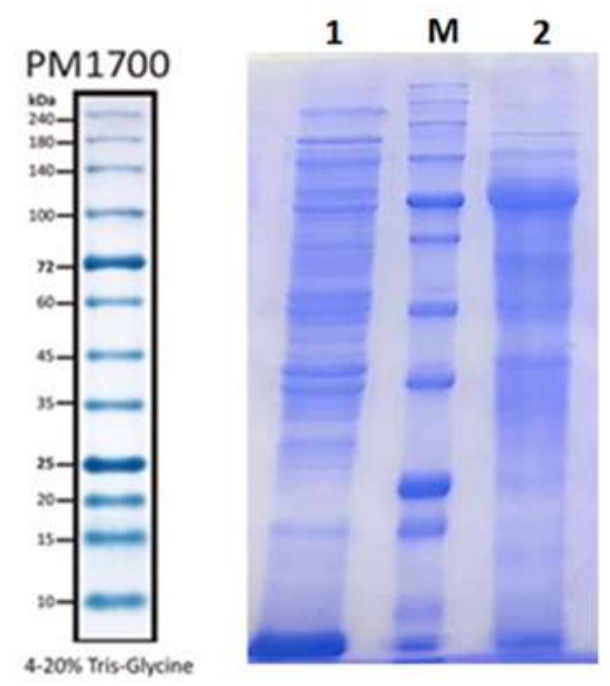

Fig. 5. Analysis of solubility of HSP70. Lane 1, bacterial supernatant (soluble phase); lane $\mathrm{M}$, protein molecular weight marker; lane 2, Bacterial deposition (insoluble phase)

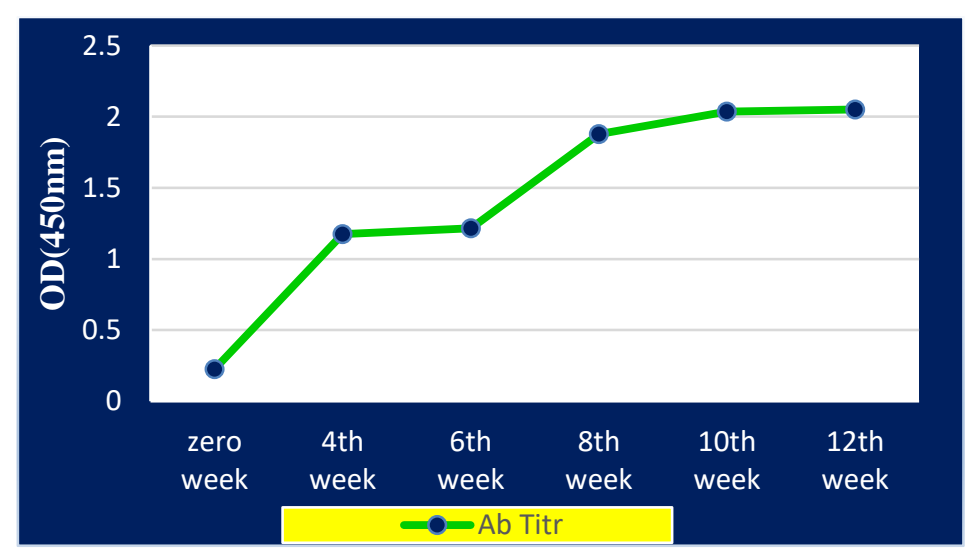

Fig. 6. Trend diagram of the increase of specific polyclonal antibody titers against a lysate of $C$. neoformans yeast in immunized rabbit serum using the ELISA technique 
The antibodies from the serum of the rabbit immunized with rHSP70 recognized the HSP70 protein in the lysate of C. neoformans yeast in ELISA. The antibody titers reached the highest levels after the third booster injection. The antibody titer reached the plateau after that [Fig. 6]. The results confirmed the desirable immunogenicity of the rHSP70.

\section{DISCUSSION}

Cryptococcosis is an exacerbating factor in patients with HIV infections and other immunocompromised patients that require proper treatment as soon as possible [9]. Diagnostic methods, whether direct or indirect, have some limitations, including false-negative results in the early stages of infection, the time-consuming culture of the agent, and falsepositive results, e.g., latex agglutination test with some bacteria and detergents $[10,2]$. As a result, the diagnosis of this infection requires more specific and sensitive techniques for faster diagnosis. Serology can provide the fastest diagnosis for this fungi infection [8].

Recent studies in several pathogen microbes have shown that members of the HSP family containing HSP70 are of significant antigenicity effect. Furthermore, HSPs are often the primary immunologic target for the humoral and cellular immune responses $[13,14]$. In $C$. neoformans yeasts, the HSP70 protein enters the plasma membrane before being released into the extracellular space by the vesicular secretory system and in association with the capsular network [12-15]. The HSP70 is an immunodominant antigen capable of triggering the humoral immune response and antibody production $[14,18]$.

In this study, we produced rHSP70 and demonstrated the immunogenicity of the protein. The purified HSP70 might be a suitable candidate for application in the serological diagnosis of $C$. neoformans. Serology is the technique of choice to detect cryptococcosis infection because it is fast, simple, widely available, and inexpensive. The reported sensitivity and specificity of $\operatorname{IgG}$ serology in latex agglutination and enzyme immunoassay techniques are $93 \%$ to $100 \%$ and $93 \%$ to $98 \%$, respectively. [2]. There is a need for a simple and reliable test that is not only inexpensive but also technically conceivable and applicable in most areas. Here, we showed that the HSP70 is not only readily producible in recombinant form, but also has the required properties of an antigen for application in diagnostic approaches.

This study attempted to establish an expression system which would allow the reproducible production of rHSP70 under standard conditions in E. coli. The results showed that the expression of $h s p 70$ was achievable using the pET-28a(+) expression system, and the BL21 cells were able to produce rHSP70 efficiently.

Recognition of the HSP70 available in the whole cell lysate of $C$. neoformans by antibodies in serum of the rabbit immunized with rHsp70 showed that the purified recombinant protein presents the antigenic epitopes. Moreover, the results showed that rHSP70 efficiently induced a specific antibody response in the immunized rabbit with a high titer, indicating the desirable antigenicity and immunogenicity of the recombinant protein.

The presented data represent that the $C$. neoformans HSP70 efficiently induces specific antibodies, which can easily be detected by serological assays based on rHSP70, implying an excellent potential for the establishment of a rapid $C$. neoformans yeast diagnostic assay. Thus, the rHSP70 protein presents a potential candidate to screen large populations for cryptococcosis in prevalent regions.

\section{ACKNOWLEDGMENT}

The authors are grateful to the Qom University of Medical Sciences, Iran, for supporting this study. We also thank Dr. Seyyed Jamal Alavinia for his technical assistance.

\section{CONFLICT OF INTEREST}

The authors declare that there are no conflicts of interest associated with this manuscript.

\section{REFERENCES}

1. Bose I, Reese AJ, Ory JJ, Janbon G, Doering TL. A Yeast under Cover: the Capsule of Cryptococcus neoformans, Eukaryot Cell. 2003; 2 (4):655-63.

2. Maziarz EK, Perfect JR. Cryptococcosis, Infect Dis Clin North Am. 2016; 30 (1): 179-206.

3. Denham ST, Brown JCS. Mechanisms of Pulmonary Escape and Dissemination by Cryptococcus neoformans, J Fungi. 2018; 4 (1): 1-17.

4. Rohatgi S and Pirofski LA. Host immunity to Cryptococcus neoformans, Future Microbiol. 2015; 10 (4): 565-581.

5. Srikanta D, Santiago-Tirado FH, Doering TL. (2014). Cryptococcus neoformans: Historical curiosity to modern pathogen, yeast, 31 (2): 47-60.

6. Perfect JR, Bicanic T. Cryptococcosis diagnosis and treatment: What do we know now, Fungal Genet Biol. 2015; 78: 49-54.

7. Upadhya R, Lam WC, Maybruck B, Specht CA, Levitz SM, Lodge JK. Induction of Protective Immunity to Cryptococcal Infection in Mice by a Heat-Killed, Chitosan-Deficient Strain of Cryptococcus neoformans, MBio. 2016; 7 (3): e00547-16.

8. Illnait MT, Vilaseca JC, Fernández CM, Martínez GF. Enzymelinked Immunosorbent Assay for Detection and Quantification of Cryptococcus neoformans Antigen, Memorias do Instituto Oswaldo Cruz. 2001; 96 (2): 241-245.

9. Rivet-Dañon D, Guitard J, Grenouillet F, Gay F, Ait-Ammar N, Angoulvant A, et al. Rapid diagnosis of cryptococcosis using an antigen detection immunochromatographic test, J Infect. 2015; 70 (5): 499-503.

10. Abassi M, Boulware DR, Rhein J. Cryptococcal Meningitis: Diagnosis and Management Update, Curr Trop Med Rep. 2015; 2 (2): 90-99.

11. Saha DC, Xess I, Zeng WY, Goldman DL. Antibody responses to Cryptococcus neoformans in Indian patients with cryptococcosis, Med Mycol. 2008; 46: 457-463. 
12. Alspaugh JA. (2015). Virulence Mechanisms and Cryptococcus neoformans pathogenesis, Fungal Genet Biol. 2015; 78: $55-58$.

13. Asea AA, Kaur P. HSP70 in Human Diseases and Disorders. In: Asea AA, Cadderwood SK, series editors. Heat shock protein, Vol 14. Switzerland: Cham; Springer. 2018. 1-394.

14. Eastman AJ, He X, Qiu Y, Davis MJ, Vedula P, Lyons DM, et al. Cryptococcal Heat Shock Protein 70 Homolog Ssa1 Contributes to Pulmonary Expansion of Cryptococcus neoformans during the Afferent Phase of the Immune Response by Promoting Macrophage M2 Polarization, J Immunol. 2015; 194 (12): 5999-6010.

15. Silveira CP, Piffer AC, Kmetzsch L, Fonseca FL, Soares DA, Staats CC, et al. The heat shock protein (Hsp) 70 of Cryptococcus neoformans is associated with the fungal cell surface and influences the interaction between yeast and host cells, Fungal Genet Biol. 2013; 60: 53-63.
16. Rodrigues ML, Nakayasu ES, Oliveira DL, Nimrichter L, Nosanchuk JD, Almeida IC, et al. Extracellular Vesicles Produced by Cryptococcus neoformans Contain Protein Components Associated with Virulence, Eukaryot Cell. 2008; 7 (1): 58-67.

17. Young M, Macias S, Thomas D, Wormley FL Jr. A Proteomic-based Approach for the Identification of Immunodominant Cryptococcus neoformans Proteins, Proteomics. 2009; 9 (9): 2578-88.

18. Kakeya H, Udono H, Maesaki S, Sasaki E, Kawamura S, Hossain MA, et al. Heat shock protein 70 (hsp70) as a major target of the antibody response in patients with pulmonary cryptococcosis, Clin Exp Immunol. 1999; 115 (3): 485-90.

19. Khalifeh-Gholi M, Kalali B, Formichella L, Göttner G, Shamsipourd F, Zarnani AH, et al. Helicobacter pylori FliD protein is a highly sensitive and specific marker for serologic diagnosis of H. pylori infection, Int J Med Microbiol. 2013; 303 (8): 618-623.

\section{Cite this article:}

Safaei Sh, Rezvan H, Fateh R, Khalife-Gholi M. Immunogenicity of the Recombinant Cryptococcus neoformans HSP70, a Potential Candidate for Developing an ELISA Kit. J Med Microbiol Infect Dis, 2020; 8 (2): 60-64. DOI: 10.29252/JoMMID.8.2.60 